\title{
A new method for measuring aerosol nebulizer output using radioactive tracers
}

\author{
A.A. Gatnash*, S.T. Chandler+, C.K. Connolly*
}

A new method for measuring aerosol nebulizer output using radioactive tracers. A.A. Gatnash, S.T. Chandler, C.K. Connolly. OERS Journals Ltd 1998.

ABSTRACT: Reproducibility and comparability of bronchial challenge tests depends critically on accurate assessment of nebulizer output. Evaporation during nebulization means that simple weighing is inaccurate, overestimating the delivered dose of active ingredient. We wanted to quantify this effect in the context of intermittent nebulization, using a dosimeter as used in bronchial provocation tests.

Output of three types of nebulizer, from the MEFAR dosimeter, was measured by radioactive tracer, using a standard solution of technetium-99m-pertechnetate $(1.5$ $\left.\mathrm{kBq} \cdot \mathrm{mL}^{-1}\right)$ in $4 \mathrm{~mL}$ of normal saline. The aerosol was impacted by suction onto a microfilter, and the radioactivity measured. Nebulizers were weighed before and after nebulization. Ratio of nebulized volume calculated from the radioactivity on the filter, to the total volume loss by weight, was expressed as nebulized ratio. The effect on output of two concentrations of methacholine, two tracers of different weights, and change in temperature, were assessed.

Nebulized ratio varied between $44.1-71.6 \%$. Results were more consistent within the same type of nebulizer than between different makes. Neither changes in molar concentration nor molecular weight affected nebulizer output or nebulized ratio.

Mean nebulized ratio was $\mathbf{5 8 . 5 \%}$, showing that calibration by weighing, overestimates the delivered dose by a factor of approximately two. Measuring radioactivity eluted from a microfilter, onto which nebulized output had been impacted proved to be a satisfactory method of calibration.

Eur Respir J 1998; 12: 467-471.
*Depts of Medicine, Memorial Hospital, Darlington and University of Newcastle upon Tyne, UK. +Dept of Medical Physics, Memorial Hospital, Darlington, UK.

Correspondence: C.K. Connolly

Memorial Hospital

Hollyhurst Road

Darlington

Co. Durham DL3 6HX

UK

Fax: 441325743435

Keywords: Bronchial lability

calibration

molar concentration

molecular weight

nebulizer

radioisotopes

Received: June 291995

Accepted after revision November 151996
Bronchial challenge tests using sequential doses of nebulized substances are used in the assessment of individual asthmatics and in epidemiological studies. Reproducibility and comparability between centres depends critically on the accurate calibration of nebulizer output. Many other variables have to be considered, such as patient cooperation, inhalation time and particle size. RYAN et al. [1] has suggested that variations in output are critical in determining differences in the results of aerosol provocation tests.

The simplest method of measuring aerosol output is by measuring weight loss. This may overestimate aerosol output because of coincidental weight loss due to evaporation. The alternative is to measure the solution actually nebulized, collecting it by impaction onto a filter. Evaporation precludes the use of weight gain but elution with the use of a chemical tracer has been shown to be satisfactory [2]. The use of a radioactive tracer may be less cumbersome, provided proper facilities are available. We wanted to assess the impact of evaporation on nebulizer output in the context of intermittent nebulization by dosimeter as used in bronchial challenge studies. We have tested and present a new method using a radioactive tracer, which we believe is more accurate than direct weighing. We have used this to assess the likely impact of evaporation on the delivered dose from intermittent nebulization.
Materials and methods

\section{Dosimeter and nebulizers}

The MEFAR Dosimeter MB3 (MEFAR, Brescia, Italy) was used. Characteristics and performance have been reported elsewhere [3]. The dosimeter pneumatic system delivered an output pressure of $88.2-99.3 \mathrm{kPa}$ at an airflow of $0.37-0.42 \mathrm{~L} \cdot \mathrm{s}^{-1}$. Three nebuliser models were used: 1) A1-A6 MEFAR, median diameter $2.0 \mu \mathrm{m}$; 2) G1-G2 Micro-Neb III (Lifecare Market Harborough, UK), median diameter $2.8 \mu \mathrm{m}$ [4]; and 3) B1-B2 Sidestream (Medic-aid Bognor Regis, UK), median diameter $2.3 \mu \mathrm{m}$ [4].

Inhalation time, pause time and the number of inhalations were kept constant. Each run consisted of 20 nebulizations of $1 \mathrm{~s}$ with an $8 \mathrm{~s}$ pause time.

\section{Nebulizer solution}

A standard solution ( $4 \mathrm{~mL}$ ) was dispensed into each nebulizer. This consisted of technetium (Tc) 99m-pertechnetate $\left({ }^{99} \mathrm{mcO}_{4}\right)($ molecular weight $(\mathrm{MW}) 163)$ diluted in normal saline at a concentration of $1.5 \mathrm{kBq} \cdot \mathrm{mL}^{-1}$. 
In order to assess the effect of the different molecular weight of the radioactive tracer on measured nebulizer performance, ${ }^{99 \mathrm{~m} T c}$-disodium oxidronate (HDP; MW 223) and 99mTc-diethylene triamine pentaacetic acid (DTPA; MW 492) were used as alternatives to the $99 \mathrm{mcO}_{4}$.

In the assessment of the effect of different drug concentrations on output, methacholine (MW 196) was added in two strengths $\left(0.003 \mathrm{mg} \cdot \mathrm{mL}^{-1}\right.$ and $\left.1.6 \mathrm{mg} \cdot \mathrm{mL}^{-1}\right)$ to the standard ${ }^{9} \mathrm{~m} \mathrm{TcO}_{4}$ solution.

\section{Aerosol collection}

The nebulizers were connected to a t-piece leading directly to a filter, without interposition of face-masks or mouthpieces, as shown in figure 1 . The aerosol was impacted onto the microfilter (Whatman glassfibre (GF/A), Whatman Maidstone, UK) connected to a suction machine rated at $10-14 \mathrm{~L} \cdot \mathrm{min}^{-1}[2]$, which was adequate for total impaction of output radioactivity with no breakthrough. This was confirmed by a second filter connected in series with the first, which was checked for the presence of any radioactivity.

\section{System loss detection}

The whole system was imaged in front of a gamma camera (IGE Starport 400AT IGE Medical, Radlett, UK) before, during and after the nebulization process, using a higher activity of $99 \mathrm{mcO}_{4}\left(10 \mathrm{MBq} \cdot \mathrm{mL}^{-1}\right)$, to establish the distribution of radioactivity within the system.

\section{Temperature}

The temperature was measured by a digital thermometer after each 20 nebulizations.

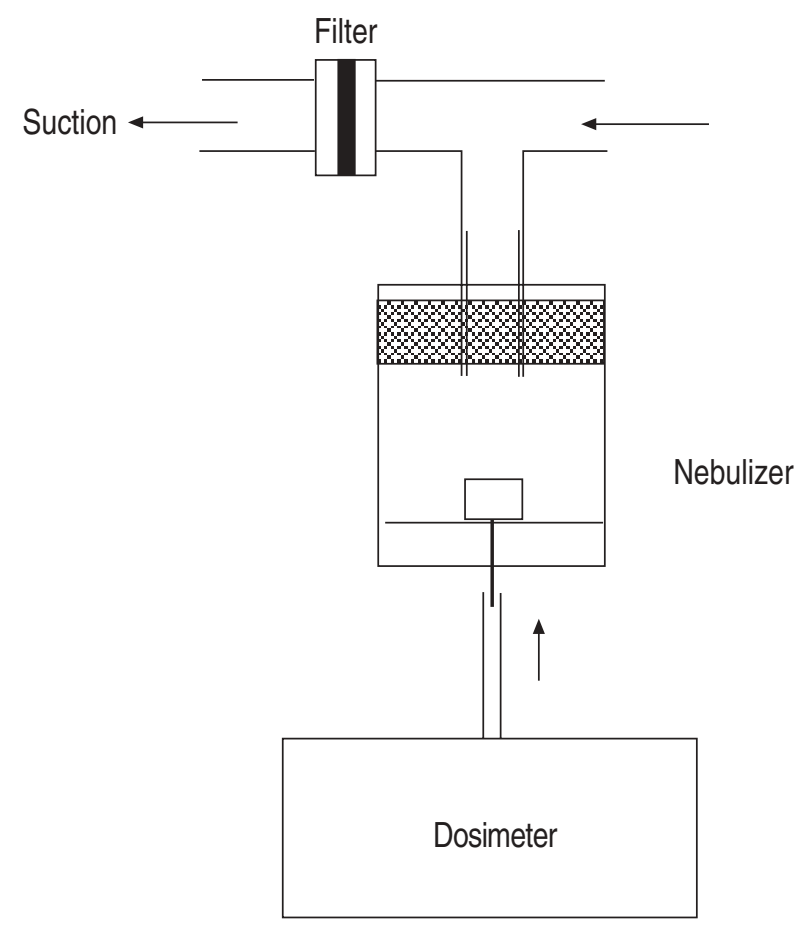

Fig. 1. - Diagram of the system for each of the nebulizers used.

\section{Output measurements}

Weight output. Each nebulizer was weighed on a precision scale (Oertling R42; London, UK) before and after each run of 20 nebulizations. Weight difference (mean of three readings) is expressed as $\mu \mathrm{g} \cdot$ nebulization $^{-1}$.

Isotopic output. The filter was changed after each run of 20 nebulizations. Radioactivity in the filter paper was eluted in a counting tube by adding a fixed volume of 5\% ethylenediamine tetraacetic acid (EDTA) solution to provide constant measurement geometry. Each tube was then counted in a gamma counter (Canberra Packard 5550; Pangbourne, UK) against a fixed volume of the standard solution. The activity was corrected for time and decay and expressed as counts. $\mathrm{min}^{-1}$. The sensitivity of the gamma counter for ${ }^{9} \mathrm{~m} \mathrm{Tc}$ is $60 \%$, giving $6 \times 10^{4}$ counts $\cdot \mathrm{min}^{-1} \mathrm{kBq}^{-1}$. The range of measurements obtained in 20 nebulizations gives 3,600-15,000 counts $\cdot \mathrm{min}^{-1}$ for the filters, against a background of 40 counts $\cdot \mathrm{min}^{-1}$. A 5 min count time gives an SD for the individual measurements of $0.4-1.3 \%$. The experimental error arising from pipetting was estimated at $<5 \%$. The overall theoretical precision of the measurement technique is $5.2 \%$. The samples were counted only once due to radioactive decay. Output $\left(\mu L \cdot\right.$ nebulization $\left.^{-1}\right)$ was calculated from the activity in the filter paper eluate against activity of the standard.

Nebulized ratio (NR). The proportion (\%) of output actually nebulized was calculated from the following formula:

$$
100 \times \text { isotopic output/weight output }
$$

Concentration change. Forty nebulizations were performed with three different nebulizers. Two samples $(1 \mathrm{~mL}$ each) were withdrawn from the residual nebulizer solution and counted against the standard. Percentage change in concentration was calculated from the formula:

$100 \times($ final activity - standard activity)/standard activity

The process was repeated three times for nebulizers A1 and B1, and twice for nebulizer G1.

Effect of different materials. The effect of different radioactive tracers with a higher molecular weight on measured nebulizer output was assessed, using 99mTc-HDP and 99mTc-DTPA. Similarly, aerosol output was measured using ${ }^{99} \mathrm{TcO}_{4}$ but with methacholine added to the nebulizer at low $\left(0.003 \mathrm{mg} \cdot \mathrm{mL}^{-1}\right)$ and high $\left(1.6 \mathrm{mg} \cdot \mathrm{mL}^{-1}\right)$ concentrations.

Reproducibility. The accuracy of the method in measuring the output from individual nebulizations was assessed by performing $10 \times 1 \mathrm{~s}$ nebulizations and changing the filter after each nebulization. This was repeated for an individual nebulizer (B1) on four separate occasions.

\section{Results}

\section{System loss}

There was no radioactivity on the second filter in the series. No loss through the air input tube was demonstrated by imaging during nebulization. The activity within 
the system, other than in the nebulizer chamber or on the first filter, amounted to $<2 \%$ of the total activity. The gamma camera image of the system of the MEFAR nebulizer, both before and after removal of the nebulizer and filter, is shown in figure 2.

\section{Temperature change}

The temperature changes with sequential nebulizations are shown in figure 3 . The mean temperature in the laboratory was $23^{\circ} \mathrm{C}$. Relative humidity was not measured at the time of each run, but varied between $40-50 \%$. The fall was $0.4^{\circ} \mathrm{C}$ at 20 and $0.75^{\circ} \mathrm{C}$ at 40 nebulizations, and did not fall $>2.4^{\circ} \mathrm{C}$ below the initial temperature, with stable readings from 140-200 nebulizations.

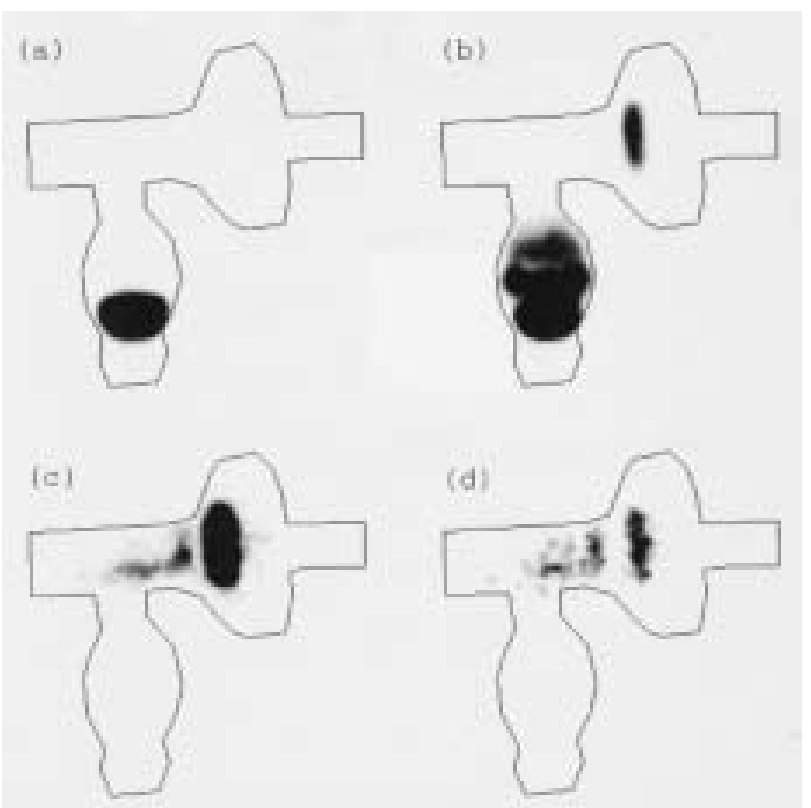

Fig. 2. - Gamma camera image of the nebulizer system: a) before nebulization; b) After 20 nebulizations; c) Nebulizer removed and image enhanced; and d) Filter removed showing minimal residual activity in the system.

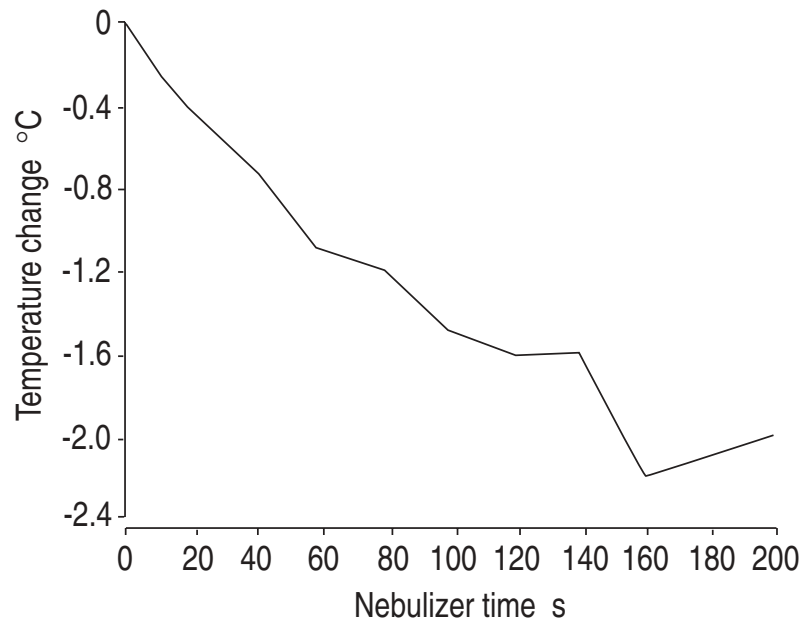

Fig. 3. - Temperature changes with repeated nebulizations.

\section{Output measurements}

The weight loss and isotopic output for the different models of nebulizers are shown in table 1 . These results summarize all the values obtained, including those made with different radioactive tracers and methacholine concentrations.

Nebulized ratio (NR) varied between 44.1-71.6\% (mean $58.5 \%$ ) according to the nebulizer used. One MEFAR nebulizer gave a consistently lower NR than the other five, with the other two pairs of nebulizers giving consistent results for the same type.

Reproducibility. The results for four repeat measurements of output for 10 separate $1 \mathrm{~s}$ nebulizations were: 4.80 (SD $0.17), 4.72(0.18), 4.73(0.19)$ and $4.61(0.10) \mu \mathrm{L}$. These results suggest that a high degree of consistency is possible with this technique and hence variations in measured values are likely to be due to individual nebulizer characteristics.

Change in concentration. The changes in concentration are shown in figure 4 . The range was $0.8-3.3 \%$ (mean $1.67 \%$ ). This is consistent with the deficit in NR being due to evaporation, after allowing for experimental error and the scatter of small quantities of solution within the system.

Effect of different materials. The effect of different materials on isotopic output are shown in table 2 . Changes in the

Table 1. - Weight loss and isotopic output

\begin{tabular}{|c|c|c|c|c|}
\hline $\begin{array}{l}\text { Neb } \\
\text { No. }\end{array}$ & $\begin{array}{l}\text { No. of } \\
\text { runs }\end{array}$ & $\begin{array}{l}\mu \mathrm{L} \cdot \text { neb- }^{-1} \\
\text { isotope }\end{array}$ & $\begin{array}{l}\mu g \cdot \text { neb }^{-1} \\
\text { weight }\end{array}$ & $\begin{array}{l}\text { Nebulized } \\
\text { ratio }\end{array}$ \\
\hline$\overline{\mathrm{A} 1}$ & 8 & $7.1(0.7)$ & $11.2(0.7)$ & $63.2(9.8)$ \\
\hline A2 & 16 & $6.0(0.8)$ & $9.2(0.6)$ & $65.0(5.6)$ \\
\hline A3 & 10 & $5.1(1.0)$ & $8.4(0.5)$ & $61.1(15.3)$ \\
\hline A4 & 12 & $8.3(0.9)$ & $11.9(1.8)$ & 70.9 (12.3) \\
\hline A5 & 12 & $5.1(0.2)$ & $10.9(0.9)$ & $46.8(4.6)$ \\
\hline A6 & 8 & $8.7(0.4)$ & $12.2(0.6)$ & $71.6(3.7)$ \\
\hline G1 & 16 & $2.4(0.2)$ & $4.0(0.2)$ & $59.7(4.7)$ \\
\hline $\mathrm{G} 2$ & 4 & $2.1(0.1)$ & $3.9(0.4)$ & $54.0(2.2)$ \\
\hline B1 & 16 & $2.0(0.4)$ & $4.1(0.5)$ & $48.3(5.9)$ \\
\hline B2 & 4 & $2.1(0.2)$ & $4.7(0.1)$ & $44.1(5.2)$ \\
\hline
\end{tabular}

Data are presented as means (SD). neb: nebulizer; A: MEFAR; B: Sidestream; G: Micro-Neb III.

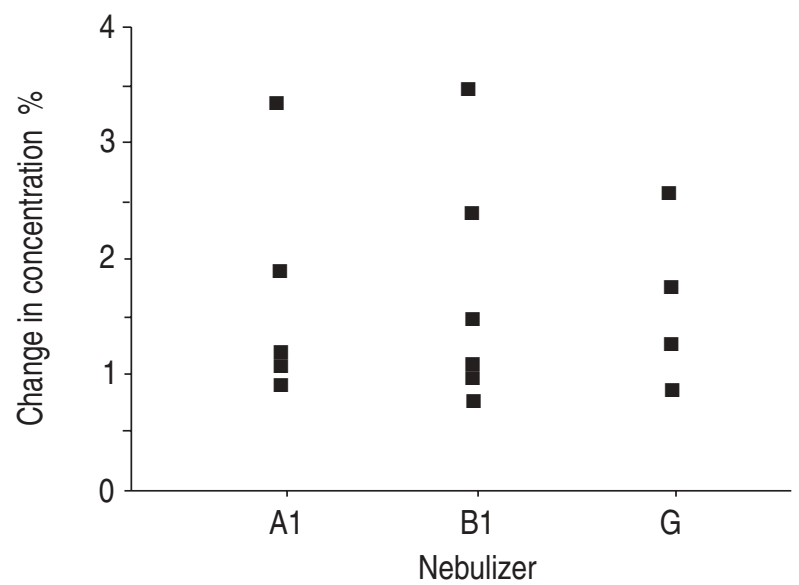

Fig. 4. - Changes in concentration of three individual nebulizers after 40 nebulizations. A1: MEFA $(3 \times 2$ samples); B1: Sidestream $(3 \times 2$ samples); G: Micro-Neb III ( $2 \times 2$ samples). 
Table 2. - Effect of different materials on isotopic output $\left(\mu \mathrm{L} \cdot \mathrm{neb}^{-1}\right)$

\begin{tabular}{|c|c|c|c|c|c|}
\hline \multirow{2}{*}{ Nebulizer } & \multirow[t]{2}{*}{${ }^{99} \mathrm{mcCO}_{2}$} & \multicolumn{2}{|c|}{ Methacholine concentration ${ }^{+}$} & \multirow[t]{2}{*}{ HDP } & \multirow[t]{2}{*}{ DTPA } \\
\hline & & Low & High & & \\
\hline A2 & $5.9(0.1)$ & $5.9 \quad(0.2)$ & $5.7 \quad(0.1)$ & $5.8 \quad(0.2)$ & $5.6 \quad(0.3)$ \\
\hline G1 & $2.3 \quad(0.1)$ & $2.8 \quad(0.2)$ & $2.6(0.7)$ & $2.4(0.6)$ & $2.3(0.1)$ \\
\hline B1 & $1.9(0.3)$ & $2.1 \quad(0.1)$ & $2.0 *$ & $2.1 \quad(0.6)$ & $1.7 \quad(0.7)$ \\
\hline Normalized for all 3 & $1.00(0.09)$ & $1.05(0.25)$ & $1.04(0.07)$ & $1.07(0.13)$ & $0.95(0.19)$ \\
\hline
\end{tabular}

Results are presented as mean (SD). A2: MEFAR; G1: Sidestream; B1: Micro-Neb III; ${ }^{99 m T c O}$ : : 99mTc-pertechnetate (molecular weight (MW) 163); HDP: 99mTc-disodium oxidronate (MW 223); DTPA: 99mTc-diethylene triamine pentaacetic acid (MW 492). +: methacholine (MW 196) at low (0.003 $\left.\mathrm{mg} \cdot \mathrm{mL}^{-1}\right)$ and high $\left(1.6 \mathrm{mg} \cdot \mathrm{mL}^{-1}\right)$ concentrations; *: only one sample obtained.

molar concentration of methacholine or molecular weight of the tracer did not give any significant difference in output from the results obtained with the standard solution of ${ }_{99} \mathrm{TcO}_{4}$ (t-test, $\mathrm{p}=0.5$ ).

\section{Discussion}

There is potential for great variation between bronchial challenge tests when performed in different centres. This makes the comparison of results difficult, particularly in large, multicentre epidemiological studies, unless standardization is consistent. Potential causes of variation are the methodology used; in particular, tidal breathing or dosimeter method, nebulizer output volume, particle size, and the ventilatory condition and pattern of breathing of the subject. Of these factors, the actual volume nebulized is probably the most important. RYAN et al. [1] reported that nebulizer output volume, but not particle size in the range 1.3-3.6 $\mu \mathrm{m}$, altered the response in bronchial challenge tests.

Our results show that the assessment of nebulizer output by weight loss considerably overestimates the aerosol delivered to the patient. This error may be as high as 50$60 \%$ in commercially available nebulizers. We confirm the findings of DeNNIS et al. [2], who used a chemical tracer to measure actual aerosol output. The discrepancy between weight loss and direct measurement of aerosol has also previously been shown by SMYE and coworkers [5, 6]. O'CaLLaGHan et al. [7] used a spectrophotometric method to assess drug output from nebulizers, but this method requires specialized equipment and may not be sensitive enough for smaller dosimeter doses.

PHIPPS et al. [8] studied the radioaerosol delivery systems used for lung ventilation imaging. However, such techniques measure high levels of radioactivity generated from relatively long periods of continuous nebulization and would not be sufficiently sensitive to measure the relatively small output from intermittent nebulization used in challenge testing. In addition, these methods require the assessment of particle size distribution using a cascade impactor. Such equipment is not commonly available in nuclear medicine departments.

In contrast, the technique we have described can measure the output from a single nebulization using relatively low levels of radioactivity and using standard equipment. Although care is needed in the handling of any radioactive materials, the amount of radioactivity used is low and the hazard is negligible.

We did not explore the effects of the change in temperature on nebulizer output. KONGERUD et al. [9] showed an increase in mean output by approximately $23 \%$ when the temperature was increased from 19 to $24^{\circ} \mathrm{C}$. Although we did sometimes perform two runs of 20 nebulizations in rapid sequence, the findings of Kongerud et al. [9] suggest that the change of $0.75^{\circ} \mathrm{C}$ demonstrated in this study over 40 consecutive nebulizations is unlikely to have had a relevant effect on evaporation.

Nebulization itself is not associated with change in concentration of a solute, but evaporation would be expected to produce an increase in the concentration of the solution. The small change in concentration demonstrated was anticipated by a simple calculation from the observed nebulized ratio, or using the model of SMYE et al. [6]. Using a simple ionic solution, Schon and KRAEMER [10] demonstrated an increase in osmolality from 282 to $432 \mathrm{mmol} \cdot \mathrm{kg}^{-1}$ as the solution in the nebulizer chamber approached exhaustion. We have confirmed that this is not a major problem at the beginning of nebulization. As long as the nebulizer is refilled for each experiment, the error arising from the change in concentration of the active constituents is negligible, certainly within the range of concentrations used in the clinical situation.

Although particle size has been found to be less important than total nebulized output in determining variation in the results of aerosol provocation tests [1], it is a potentially confounding variable. Although we have not addressed this point, published results would suggest that drug concentration has little effect on particle size [11]. We have shown that a change in methacholine concentration from 0.003 to $1.6 \mathrm{mg} \cdot \mathrm{mL}^{-1}$, covering the range of concentrations used in our aerosol provocation tests, produced no change in nebulizer output.

Similarly, the output was independent of the molecular weight of the tracers used. These results were obtained with actual concentrations used in our bronchial provocation tests. Results obtained under different conditions, such as continuous nebulization or until the nebulizer runs dry, may well give differing results.

In therapy, continuous nebulization is used, where the dose actually delivered is often less critical than in bronchial provocation tests. Nevertheless, where the clinical dose is important, nebulizer calibration should include estimates of NR during continuous nebulization. This might be of particular relevance where a critical dose of inhaled corticosteroids is required, or with the more viscous solutions produced by antibiotics. Our results cannot be applied directly to these situations. The results do, however, emphasize the need to test the combination of delivery device and nebulizer in conditions as close as possible to those of actual usage, particularly as others have suggested that output may be modified by addition of the $\beta$-agonist fenoterol to the radioactive tracer. However, output 
measurements performed with either drug alone or only radioactive tracer, were comparable [12].

Differences in interactions between the nebulizer's plastic surfaces and other radioisotopes not tested here might also cause differences between apparently similar nebulizers or batches. It is, therefore essential that the actual nebulizer to be used should be tested when doses are critical.

The method described here represents a simple technique using standard equipment and materials available in many hospitals, and produces consistent results. It has been demonstrated that the dose delivered by the nebulizer may be overestimated by between approximately 30-60\% if calibration is achieved by simple weighing.

\section{References}

1. Ryan G, Dolovich MB, Oldenburg FA, Newhouse MT. Standardization of inhalation provocation tests: influence of nebulizer output, particle size and method of inhalation. J Allergy Clin Immunol 1981; 67: 156-162.

2. Dennis JH, Stenton SC, Beach JR, Avery AJ, Walters EH, Hendrick DJ. Jet and ultrasonic nebuliser output: use of a new method for direct measurement of aerosol output. Thorax 1990; 45: 728-732.

3. Arossa $\mathrm{W}$, et al. Assessment of the variability of physical properties of aerosols generated by a new Italian nebulizer. Ital J Chest Dis 1998; 42: 43-45.
4. Hurley PK, Smye SW. Performance assessment of a range of commercial jet nebulisers. Technol Health Care 1994; 1: 209-214.

5. Smye SW, Shaw A, Norwood HM, Littlewood JM. Some factors influencing the efficiency of a jet nebuliser system. Clin Phys Physiol Meas 1990; 11: 167-175.

6. Smye SW, Jollie MI, Cunliffe H, Littlewood JM. Measurement and prediction of drug solvent losses by evaporation from a jet nebuliser. Clin Phys Physiol Meas 1992; 13: 129-134.

7. O'Callaghan C, Clarke AR, Milner AD. Inaccurate calculation of drug output from nebulisers. Eur J Paediatr 1989; 148: 473-474.

8. Phipps P, Borham P, Gonda I, Bailey L, Bautovich G, Anderson S. A rapid method for the evaluation of diagnostic radioaerosol delivery systems. Eur J Nucl Med 1987; 13: 183-186.

9. Kongerud J, Soyseth V, Johansen B. Room temperature influences output from the Wright jet nebulizer. Eur Respir J 1989; 2: 681-684.

10. Schoni MH, Kraemer R. Osmolality changes in nebulizer solutions. Eur Respir J 1989; 2: 887-892.

11. Clay M, Pavia D, Newman SP, Clarke SW. Factors influencing the size distribution of aerosols from jet nebulisers. Thorax 1983; 38: 755-759.

12. Fuller HD, Dolovich MB, Chambers C, Newhouse MT. Aerosol delivery during mechanical ventilation: a predictive in vitro lung model. J Aerosol Med 1992; 5: 251-259. 